\title{
Self-assembled Nanoparticles of Polycationic b Rush and p Hotosensitizer for Synergistic Photothermal and Photodynamic Therapies Against Periodontitis
}

\section{Enyu Shi}

Tianjin Medical University Dental School: Tianjin Stomatological Hospital

\section{Liya Bai}

Tianjin Medical University

\section{Lujia Mao}

Tianjin Medical University Dental School: Tianjin Stomatological Hospital

\section{Hanping Wang}

Tianjin Medical University Dental School: Tianjin Stomatological Hospital

\section{Xiaoying Yang}

Tianjin Medical University

\section{Yinsong Wang}

Tianjin Medical University

\section{Mingming Zhang}

Chinese Academy of Medical Sciences \& Peking Union Medical College Institute of Biomedical

\section{Engineering}

\section{Changyi Li}

Tianjin Medical University Dental School: Tianjin Stomatological Hospital

\section{Yue Wang ( $\nabla$ wangyue1@tmu.edu.cn )}

Tianjin Medical University Dental School: Tianjin Stomatological Hospital

\section{Research}

Keywords: Nanoparticles, polycationic brush, photothermal therapy, photodynamic therapy, periodontitis

Posted Date: August 26th, 2021

DOl: https://doi.org/10.21203/rs.3.rs-832444/v1

License: (c) (1) This work is licensed under a Creative Commons Attribution 4.0 International License. Read Full License 


\section{Abstract}

Background: Periodontitis is a chronic inflammatory disease in oral cavity owing to bacterial infection. Photothermal therapy (PTT) and photodynamic therapy (PDT) have many advantages for antibacterial treatment. As an excellent photosensitizer, indocyanine green (ICG) shows prominent photothermal and photodynamic performances. However, it is difficult to pass through the negatively charged bacterial cell membrane, thus limits its antibacterial efficacy for periodontitis treatment.

Results: In this work, we developed a nanosystem from self-assembly of ICG and polycationic brush for synergistic PTT and PDT against periodontitis. A star-shaped polycationic brush, poly(2-

(dimethylamino)ethyl methacrylate) (sPDMA), was synthesized via atom transfer radical polymerization (ATRP) of DMA monomer from bromo-substituted $\beta$-cyclodextrin initiator (CD-Br). ICG was then selfassembled with sPDMA to form ICG-loaded sPDMA (sPDMA@ICG) nanoparticles (NPs), and the physicochemical properties of these NPs were characterized in detail. In vitro antibacterial effects of sPDMA@ICG NPs were evaluated in porphyromonas gingivalis (Pg), one of the recognized periodontitis pathogens, and in vivo anti-periodontitis effects of NPs were investigated in a rat periodontitis model. Benefiting from the unique brush-shaped architecture of sPDMA polycation, sPDMA@ICG NPs efficiently delivered ICG into the bacterial cells through promoting their adsorption and penetration abilities, and also exhibited effective antibacterial and anti-periodontitis actions via synergistic PTT and PDT both in vitro and in vivo.

Conclusions: This work developed a promising nano-photosensitizer for synergistic PTT and PDT for antibacterial and periodontitis treatments in clinic.

\section{Introduction}

Periodontitis is the sixth most prevalent disease in the world, with more than 743 million people worldwide at risk [1]. It is a chronic inflammatory disease caused by bacteria, which eventually leads to the formation of periodontal pockets, the loss of alveolar bone and the loosening of teeth [2, 3]. In addition, periodontal disease is reported to be related to some systemic diseases, such as arteriosclerosis, diabetes, aspiration pneumonia and vascular disease [4]. At present, mechanical debridement and antibiotics are commonly used in clinic $[5,6]$. However, in most cases, mechanical debridement is difficult to completely remove the periodontitis infection in deep periodontal pocket, furcation and irregular area of root surface $[7,8]$. Antibiotic treatment can effectively eliminate periodontal pathogens, but long-term use of antibiotics will bring many problems, such as producing drug-resistant bacteria (so-called superbacteria), dysbacteriosis, gastrointestinal diseases and so on $[9,10]$. Hence, it is necessary to develop a more effective method for periodontitis treatment.

Phototherapy, including photothermal therapy (PTT) and photodynamic therapy (PDT), is widely recognized as an excellent non-invasive approach for cancer ablation and also has been used for antimicrobial treatment in recent years. PTT is a hyperthermia therapeutic method that applies near- 
infrared (NIR) light-absorbing agents to exert a lethal temperature elevation effect on bacteria under laser irradiation [11]. PDT can induce the generation of cytotoxic reactive oxygen species (ROS) to bring about the fatal and extensive damages to bacteria through the oxidation of nucleic acids, lipids and proteins [12-14], based on the photochemical reaction that involves three basic components (photosensitizer, molecular oxygen and laser at a particular wavelength). These phototherapeutic methods have a high spatiotemporal selectivity for localized tissue ablation, and thus are highly safe for applications in vivo. More importantly, they are equally effective to drug-resistant and non-drug-resistant pathogenic bacteria, and will not cause bacterial resistance even if a long-term use $[15,16]$. An increasing number of investigations have been carried out for antimicrobial treatment through PTT and PDT. In a recent report [17], we developed a novel photosensitizer with excellent bacteria penetrating ability and combined PDT and antibiotics for periodontitis treatment by fighting periodontal pathogenic bacteria more efficiently.

Some NIR light-absorbing agents exhibit prominent photothermal and photodynamic performances simultaneously. Indocyanine green (ICG) is a highly safe NIR light-absorbing agent and has been approved by the Food and Drug Administration (FDA) for clinical use since 1959. In recent decade, it has been widely applied for cancer treatment via synergistic PTT and PDT $[14,18]$. However, because of its negative charges and water solubility, ICG is difficult to pass through the bacterial cell membrane that is also negatively charged and characterized by lipid bimolecular structure. Gram-negative bacteria have the inner and the outer membranes composed of phospholipids and lipopolysaccharide, and thus it is more difficult for ICG to penetrate both these bacterial membranes. In addition, as a natural barrier from external dangers, the bacterial biofilm further prevents the penetration of ICG. All these limitations will be unfavorable for fully exerting the PTT and PDT performances of ICG against pathogenic bacteria.

Some cationic polymers exhibit strong bacterial cell penetration activity, mainly manifested their adsorption onto the bacterial surfaces with negative charges $[19,20]$, and even interaction with the inner membranes of gram-negative bacteria [21, 22]. Polycationic molecular brushes are a novel kind of branched cationic polymers that is defined as dense layers of cationic polymer chains grafted on a molecule. Compared to linear or ordinary branched cationic polymers, when a polymer is in brush region, the distance $(D)$ between the neighboring chains is less than twice of gyration radius $\left(R_{g}\right)$ of a free polymer chain $\left(D / R_{g}<2\right)$. With such high grafting density, the chains are forced to stretch out due to electrostatic repulsion and the geometrical constraint. And therefore, the stability of carriers constructed by the brush can be improved due to the high osmotic repulsion within the brush layer. Besides, the working concentration can also be decreased, since the densely positive chains provide significant binding capability with the negatively charged molecules. Polycationic brushes have many biomedical applications [23], especially in gene delivery systems [24, 25]. Polycationic brushes can condense genes to form complexes, and thus improve the cellular uptakes and transfection efficiencies of these genes. Furthermore, polycationic brushes often exhibit higher gene transfection performance and less cytotoxicity than the linear ones with comparable molecular weights [26-28]. Combination these advantages, we believe that polycationic brushes being used as a carrying material can facilitate 
transporting ICG into the bacteria cells through interaction with bacterial membrane and biofilm, and hence promoting the PTT and PDT performances of ICG to kill the bacteria.

In this paper, a self-assembled nanosystem of ICG and polycationic brushes was developed for synergistic PTT and PDT against periodontitis (Scheme 1). A star-shaped cationic brush, poly(2(dimethylamino)ethyl methacrylate) (sPDMA), was first synthesized via atom transfer radical polymerization (ATRP) of DMA monomer from bromo-substituted $\beta$-cyclodextrin initiator (CD-Br). ICG was then self-assembled with the SPDMA brush in aqueous solution to form ICG-loaded SPDMA (sPDMA@ICG) nanoparticles (NPs), and the physicochemical properties of the NPs were characterized in detail. In vitro antibacterial activities of sPDMA@ICG NP-mediated synergistic PTT and PDT were evaluated in Porphyromonas gingivalis $(\mathrm{Pg})$, one of the recognized periodontitis pathogens [29, 30]. An animal model of periodontitis was further constructed in Sprague-Dawley (SD) rats for evaluation of in vivo anti-periodontitis effects of synergistic PTT and PDT mediated by sPDMA@ICG NPs. The results demonstrated that benefiting from the unique brush-shaped architecture of SPDMA polycation, sPDMA@ICG NPs exhibited effective antibacterial and anti-periodontitis actions via synergistic PTT and PDT both in vitro and in vivo.

\section{Materials And Methods}

\section{Materials}

ICG was purchased from J\&K Scientific Ltd. (Beijing, China). 2-(Dimethylamino) ethyl methacrylate (DMA) and 2-bromoisobutyryl bromide (BiBB) both with a purity of approximately $98 \%$ were bought from SigmaAldrich (Milwaukee, USA), and DMA was passed through a basic alumina column before use to remove polymerization inhibitor. $\beta$-Cyclodextrin ( $\beta$-CD) and 2,2-bipyridine (Bpy) were supplied by Sinopharm Chemical Regent Co., Ltd. (Shanghai, China). Anhydrous N-methyl-2-pyrrolidone (NMP) was obtained from Alfa Aesar (Ward Hill, MA, USA). Cuprous chloride ( $\mathrm{CuCl}$ ) was dissolved in concentrated hydrochloric acid, precipitated via dilution with water, washed separately with ethanol and ethyl ether, and finally vacuum dried in an oven. Copper chloride $\left(\mathrm{CuCl}_{2}\right)$ was baked at $120^{\circ} \mathrm{C}$ to remove the crystal water before use. 2,7-Dichlorodihydro fluorescein diacetate (DCFH-DA), N-phenyl-1-naphthylamine (NPN), 4', 6diamidine-2-phenylindole dihydrochloride (DAPI), and hematoxylin \& eosin (H\&E) were all purchased from Sigma-Aldrich. Singlet Oxygen Sensor Green Reagent (SOSG), Cell Counting Kit-8 (CCK-8), and the LIVE/DEAD BacLight Bacterial Viability Kit were purchased from Thermo Fisher Scientific (Waltham, MA, USA).

\section{Cells and animals}

The strain of Pg (code BNCC 337441), sourced from BeNa Culture Collection (Beijing, China), was cultured in the Brain Heart Infusion (BHI) Broth or the Columbia blood agar plates (Solarbio, Beijing, China) at $37^{\circ} \mathrm{C}$ in an anaerobic chamber with an atmosphere of $80 \% \mathrm{~N}_{2}, 10 \% \mathrm{H}_{2}$ and $10 \% \mathrm{CO}_{2}$. 
Female Sprague Dawley (SD) rats with average body weight of $150 \mathrm{~g}$ were provided by Vital River Laboratory Animal Technology Co., Ltd. (Beijing, China) and were kept in plastic cages with free access to food and water. Periodontitis animal model was constructed in SD rats according to our previous method [17]. After anesthetization, the rats were ligated with orthodontic steel wires at the gingival sulcus of the left maxillary second molar and then feed with $10 \%$ sucrose water for 4 weeks to induce periodontitis. All animal experiments in this study were carried out according to the protocol approved by the Tianjin Medical University Animal Care and Use Committee.

\section{Preparation and characterization of sPDMA@ICG NPs}

$\mathrm{CD}-\mathrm{Br}$ was firstly synthesized via the esterification of $\beta-\mathrm{CD}$ with $\mathrm{BiBB}$ according to the previous work [23] and then Polycationic brush sPDMA was synthesized via atom transfer radical polymerization (ATRP) of DMA monomer using CD-Br as an initiator. The detailed methods were described in the supporting information.

Next, an improved nanoprecipitation method was used to prepare sPDMA@ICG NPs. Briefly, ICG (5 mg) was dissolved in $100 \mu \mathrm{L}$ of dimethyl sulfoxide (DMSO) as a stock solution, and meanwhile $14 \mathrm{mg}$ of sPDMA was dissolved in $10 \mathrm{~mL}$ deionized water. After that, 2, 4, 8, 12, and $26 \mu \mathrm{L}$ of ICG stock solution were mixed separately with $1 \mathrm{~mL}$ of sPDMA aqueous solution and stirred for $12 \mathrm{~h}$ at room temperature in the dark, thus obtaining sPDMA@ICG NPs with different ICG contents. The ultraviolet-visible-near infrared (UV-Vis-NIR) absorption spectra of these sPDMA@ICG NPs were recorded under a U-3310 spectrophotometer (Hitachi High-Tech, Tokyo, Japan). Here, $8 \mu \mathrm{L}$ of ICG stock solution was diluted with 1 $\mathrm{mL}$ of deionized water to be used as the control. The diameters and polydispersity indexes (PDIs) of these NPs were detected using an automatic particle size detector (Zetasizer Nano ZS, Malvern, UK).

By comprehensive consideration, the ICG/sPDMA weight ratio of 4/14 was chosen to prepare sPDMA@ICG NPs using the same method as above. The thus-obtained nanoparticle dispersion was transferred into a dialysis bag (MWCO $500 \mathrm{Da}$ ) and dialyzed against deionized water to remove DMSO and unassembled ICG. Finally, the morphology of sPDMA@ICG NPs was characterized under a HT7700 transmission electron microscope (TEM) (Hitachi High-Tech, Tokyo, Japan) and their size and size distribution were further detected.

\section{Statistical analysis}

All the experiments were carried out independently at least three times. The experimental data were presented as mean \pm standard deviation (SD). Statistical analysis was performed by one-way analysis of variance (ANOVA) and $P<0.05$ was considered to be significant statistically.

\section{Results And Discussion}

\section{Preparation and characterization of sPDMA@ICG NPs}


A star-shaped polycation sPDMA was first synthesized via ATRP of DMA monomer from CD-Br initiator according to our previous works $[23,31]$, and the synthesis route is shown in Fig. S1. From the ${ }^{1} \mathrm{H}$ NMR spectrum of CD-Br initiator in Fig. S2, the number of $\mathrm{Br}$ per $\beta$-CD molecule was calculated to be 7 , indicating that 7 PDMA chains were grafted from one $\beta$-CD. After polymerization, the characteristic peaks representing protons in PDMA appeared at 4.1, 2.6 and 2.3 ppm (Fig. 1a), proving the success of polymerization. Since the content of $\beta-C D$ in the polymer was relatively small, the proton signals of $\beta-C D$ were not observed in the spectrum. According to the polymerization yield, the degree of polymerization $\left(D_{p}\right)$ was estimated to be about 12.2 per chain and the molecular weight of SPDMA was approximately 15,600 . The molecular weight distribution $\left(M_{w} / M_{n}\right)$ was 1.24 characterized by gel permeation chromatography. To validate whether the polymer is in molecular brush regime, the gyration radius $\left(R_{g}\right)$ and the distance $(D)$ between the neighboring chains were estimated. According to the following equation [32], $R_{g}=0.5 D_{p}^{0.5}, R_{g}$ of PDMA chain was calculated to be about $1.75 \mathrm{~nm}$. While $D$ was estimated to be less than the outer diameter of $\beta-C D(1.54 \mathrm{~nm})$ [33]. Thus, $D / R_{g}$ would be less than 0.88 , meaning that the PDMA chains are in molecular brush regime. With such high grafting density, the PDMA chains will be forced into a stretched state due to the steric constraint, endowing SPDMA unique properties different from its random coil state.

sPDMA@ICG NPs were prepared from self-assembly of ICG and sPDMA in aqueous solution using the nanoprecipitation method described in the Supporting information. Fig. S3 shows the ultraviolet-visiblenear infrared (UV-Vis-NIR) absorption spectra of ICG and SPDMA@ICG NPs. The characteristic absorption peak of ICG at $780 \mathrm{~nm}$ showed an obvious red shift after assembled with SPDMA, indicating that ICG had entered a less polar environment. Besides, the peak intensity of ICG monomer at $780 \mathrm{~nm}$ significantly decreased, while that of ICG dimer at $700 \mathrm{~nm}$ increased a little at the same time. This demonstrated that the distance between ICG molecules is closer in the NPs than in aqueous solution according to a previous report [34]. sPDMA@ICG NPs with different weight ratios of ICG/sPDMA were further prepared using the same method. In the UV-Vis-NIR spectra of these NPs, the similar peak shapes were observed clearly (Fig. 1b), meaning that ICG was loaded in similar environment with similar forms. When the ICG/sPDMA weight ratio was 4/14, sPDMA@ICG NPs displayed the smallest particle size and the narrowest polydispersity index (PDI) (Fig. 1c). Hence, sPDMA@ICG NPs used in the following investigation were prepared at this weight ratio. Under the observation of transmission electron microscope (TEM), sPDMA@ICG NPs had a regularly spherical shape (Fig. 1d).The diameter of sPDMA@ICG NPs determined using the dynamic light scattering method was $206 \mathrm{~nm}$ with a narrow size distribution, and their zeta potential was about $+18.4 \mathrm{mV}$ (Fig. 1e). Within 5 days, the size and size distribution of sPDMA@ICG NPs changed little (Fig. 1f). In the UV-Vis-NIR absorption spectra, the peak intensities of sPDMA@ICG NPs were almost the same in this period (Fig. 1g), while free ICG showed obviously decreased absorption over time (Fig. 1h). These results demonstrate sPDMA@ICG NPs have better storage stability than free ICG.

\section{Photothermal and photodynamic performances of sPDMA@ICG NPs}


ICG is a photosensitizer with both photothermal and photodynamic performances. After assembled with sPDMA to form PDMA@ICG NPs, the photothermal and photodynamic performances of ICG were further investigated and the results are shown in Fig. 2. After irradiation with an $808 \mathrm{~nm}$ laser at $2 \mathrm{~W} / \mathrm{cm}^{2}$, sPDMA@ICG NPs showed significant temperature elevations in an ICG concentration-dependent manner and the temperatures of their solutions rapidly increased within 2 min (Fig. 2a). Moreover, sPDMA@ICG NPs had slightly higher photothermal efficiency than that of free ICG at the ICG concentration of 35 $\mu \mathrm{g} / \mathrm{mL}$ and their solution temperature increased from 22 to $55^{\circ} \mathrm{C}$ (Fig. S4A and B). It thus can be deduced that sPDMA@ICG NPs have good photothermal property. The photodynamic performance of SPDMA@ICG NPs was next evaluated by detecting the ROS generation using a green fluorescence probe, Singlet Oxygen Sensor Green (SOSG). The fluorescence intensity of SOSG at $525 \mathrm{~nm}$ for free ICG and sPDMA@ICG NPs (10 $\mu \mathrm{g} / \mathrm{mL} I C G)$ both gradually increased with the increase of irradiation time, while it was not observed in phosphate buffered saline (PBS) or sPDMA (Fig. 2b). These results confirmed the significant photodynamic performances of ICG and sPDMA@ICG NPs. By comparison, sPDMA@ICG NPs showed an obviously weaker photodynamic performance, which may be owing to the change of light absorption property of ICG after self-assembly with sPDMA.

\section{Bacterial surface adsorption and outer membrane penetration of sPDMA@ICG NPs}

ICG is negatively charged and water soluble, hence it is difficult for it to pass through the bacterial cell membrane that is also negatively charged and characterized by lipid bimolecular structure. In this study, sPDMA@ICG NPs were prepared from self-assembly of ICG and SPDMA in aqueous solution, hoping to promote the adsorption and penetration of ICG in bacterial cells by taking advantage of the high positive charge density of sPDMA. The surface adsorption of sPDMA@ICG NPs towards Pg, a Gram-negative bacterium that is directly associated with periodontitis, was firstly evaluated through monitoring the change of bacterial surface charge property after 3-hour incubation. As shown in Fig. 3a, the negative charges on the surface of Pg incubated with sPDMA@ICG NPs were neutralized continuously, reflecting in the change of zeta potential from negative to positive and the further enhanced zeta potential value as the ICG concentration increasing. But in the meantime, the zeta potential of Pg incubated with free ICG maintained negative values. The above results demonstrate that sPDMA@ICG NPs can be adsorbed onto the bacterial surfaces via electrostatic interaction.

N-phenyl-1-naphthylamine (NPN) is often applied as a fluorescence probe to detect the penetration and damage of bacterial cell membrane [35], since it emits weak fluorescence in aqueous solution but strong fluorescence after spontaneously entering the hydrophobic region of cell membranes. Herein, NPN was used as a fluorescence indicator to evaluate the penetration of sPDMA@ICG NPs through the outer membrane of $\mathrm{Pg}$, and the results are shown in Fig. 3b. NPN displayed very strong fluorescence signals in the bacteria after 3-hour incubation with sPDMA and sPDMA@ICG NPs. However, no visible increased fluorescence intensity of NPN was observed in the bacteria incubated with free ICG as compared to that incubated with PBS. It thus can be deduced that SPDMA has significant bacterial cell membranepenetrating ability due to the superhigh density of positive charges on its brush layer. Moreover, upon 808 
nm laser irradiation at $2 \mathrm{~W} / \mathrm{cm}^{2}$ for 5 min, free ICG and SPDMA@ICG NPs further enhanced the permeability of outer membrane of $\mathrm{Pg}$, demonstrating the damage of bacterial membrane caused by the photothermal and photodynamic performances of ICG.

The uptakes of free ICG and sPDMA@ICG NPs in Pg were also compared after 3-hour incubation through observing the intracellular fluorescence of ICG. Figure $3 \mathrm{c}$ shows the confocal microscopic images of the bacteria with staining of 4',6-diamidino-2-phenylindole dihydrochloride (DAPI). The intensive red fluorescence representing ICG was clearly observed in SPDMA@ICG NP-incubated Pg when the ICG concentration was $10 \mu \mathrm{g} / \mathrm{mL}$, while almost no fluorescence was observed for free ICG with the same concentration. This indicated that SPDMA@ICG NPs were efficiently accumulated in the bacterial cells after penetrating the cell membranes, and thus would facilitate ICG to exert antibacterial effects through synergistic PTT and PDT.

\section{In vitro antibacterial effects of sPDMA@ICG NPs with laser irradiation}

Since sPDMA@ICG NPs can effectively deliver ICG into the bacteria cells and exhibit synergistic PTT and PDT performances, we further evaluated the antibacterial effects of sPDMA@ICG NPs. First, the PTT effect of sPDMA@ICG NPs was examined in Pg. After incubation with sample solutions for $3 \mathrm{~h}$, the bacterial suspensions were centrifuged to remove the unbound samples and the precipitations were dispersed in PBS. These bacterial suspensions were irradiated with an $808 \mathrm{~nm}$ laser at $2 \mathrm{~W} / \mathrm{cm}^{2}$ for 10 min, and their temperatures were further recorded using an infrared thermal camera within this period. sPDMA@ICG NPs maintained high photothermal conversion efficiency in the bacterial suspension, which was significantly stronger than free ICG (Fig. $4 a$ and b). After laser irradiation for $5 \mathrm{~min}$, the temperature raised and maintained in the range of $45-50^{\circ} \mathrm{C}$, which was greatly higher than the body temperature. At this temperature range, the oral pathogenic bacteria can be damaged and inhibited effectively. This further proves that sPDMA@ICG NPs efficiently delivered ICG into the bacteria to exert the PTT efficacy against periodontitis.

PDT can induce the generation of ROS that possesses strong cytotoxicity via destroying bacterial lipids, proteins and genes. To evaluate the PDT performance of sPDMA@ICG NPs in Pg, 2',7'-

dichlorodihydrofluorescein diacetate (DCFH-DA) was used as a fluorescent probe to detect the intracellular production of ROS. When a large amount of ROS is produced in the cells, non-fluorescent DCFH-DA can be oxidized into a fluorescent product 2',7'-dichlorofluorescein (DCF) through a series of reactions. The fluorescence intensity of DCF is related directly to the intracellular level of ROS. Figure 4c compares the fluorescence intensities of DCF in the bacteria. Upon 10 minute-laser irradiation at $808 \mathrm{~nm}$ at 2 W/ $\mathrm{cm}^{2}$, free ICG and SPDMA@ICG NPs both induced the notably enhanced fluorescence intensities of DCF, indicating that they exerted the PDT performances to trigger the generations of intracellular ROS. By comparison, sPDMA@ICG NPs showed a significantly more potent PDT performance, inflecting in a higher fluorescence intensity of DCF. This further proves SPDMA@ICG NPs efficiently delivered ICG into the bacteria to exert the PDT efficacy against periodontitis. 
The antibacterial activity of sPDMA@ICG NP-mediated PTT and PDT was next evaluated in Pg using the CCK-8 assay. The influence of SPDMA on the bacterial viability was assessed firstly at different concentrations. Results in Fig. S5 shows that the bacterial viability decreased notably when the sPDMA concentration was larger than $50 \mu \mathrm{g} / \mathrm{mL}$. Therefore, the concentration of sPDMA below $25 \mu \mathrm{g} / \mathrm{mL}$ was chosen in the following study. Without laser irradiation, the bacterial viabilities in the groups of free ICG and SPDMA@ICG NPs maintained at the high levels in the ICG concentration range of $1-10 \mu \mathrm{g} / \mathrm{mL}$. After $808 \mathrm{~nm}$ laser irradiation at $2 \mathrm{~W} / \mathrm{cm}^{2}$ for $5 \mathrm{~min}$, free ICG and sPDMA@ICG NPs both inhibited the bacterial growth remarkably, but by comparison, sPDMA@ICG NPs displayed a much higher antibacterial effect (Fig. 4d). Under the TEM observation, the adsorption of sPDMA@ICG NPs was clearly visible on the surfaces of $\mathrm{Pg}$, and after laser irradiation, the bacterial membrane was ruptured and the bacterial cells were disintegrated (Fig. 4e). Colony formation assay was further applied to evaluate the antibacterial activity visibly in Pg. As shown in Fig. 4f, sPDMA@ICG NPs with laser irradiation almost completely inhibited the bacterial growth at the ICG concentration of $10 \mu \mathrm{g} / \mathrm{mL}$, while the bacteria in the other groups grew in different degrees. These results indicated that sPDMA@ICG NPs exerted synergistic PTT and PTT.

\section{Destruction effect of sPDMA@ICG NPs with laser irradiation on the plaque biofilm}

Dental plaque, which actually is the same as the bacterial biofilm, has been known to be the basis for the survival of microorganisms. The dental plaque can resist the penetration of antibacterial agents and thus reduce their antibacterial effects $[36,37]$. This shows that the destruction of dental plaque will help to antibacterial treatment. Herein, the pathogenic bacteria were extracted from the periodontitis rats and incubated in the liquid culture medium for $3 \mathrm{~d}$ to form the plaque biofilm, which was further applied for evaluation of the destruction effect of sPDMA@ICG NP-mediated PTT and PDT. Only upon laser irradiation (808 $\mathrm{nm}, 2 \mathrm{~W} / \mathrm{cm}^{2}$ and $5 \mathrm{~min}$ ), free ICG and SPDMA@ICG NPs remarkably destroyed the plaque biofilms at the ICG concentration of $10 \mu \mathrm{g} / \mathrm{mL}$ (Fig. 5a). Not surprisingly, sPDMA@ICG NPs with laser irradiation exhibited a more potent destruction effect on the plaque biofilm than free ICG with laser irradiation, reflecting in a larger area $\left(2.87 \mathrm{~cm}^{2}\right)$ of damaged biofilm (Fig. $5 \mathrm{~b}$ ). These damaged plaque biofilms were further stained with the LIVE/DEAD (SYTO9/PI) BacLight Bacterial Viability Kit to evaluate the antibacterial activity. Under a fluorescence microscope, the live and dead bacteria emitted green and fluorescence separately. Similar to the above results, the bacteria in the group of sPDMA@ICG NPs with laser irradiation were almost completely killed (Fig. 5c). These results suggest that synergistic PTT and PDT mediated via sPDMA@ICG NPs is a promising antibacterial method for periodontitis treatment because of its destruction effect on the plaque biofilm and penetration ability through the bacterial membrane.

\section{In vivo PTT and PDT performances of sPDMA@ICG NPs}

A periodontitis animal model was constructed in Sprague Dawley (SD) rats according to our previous method [17]. After anesthetization, the rats were ligated with orthodontic steel wires at the gingival sulcus of the left maxillary second molar and then feed with $10 \%$ sucrose water for 4 weeks to induce 
periodontitis. These rats were given the treatments of sPDMA, free ICG and sPDMA@ICG NPs alone and their combination with laser irradiation. Here, $50 \mu \mathrm{L}$ of sample solutions containing $20 \mu \mathrm{g} / \mathrm{mL}$ of ICG and/or $70 \mu \mathrm{g} / \mathrm{mL}$ of sPDMA were administrated via smear and laser irradiation was carried out at 808 $\mathrm{nm}$ at $2 \mathrm{~W} / \mathrm{cm}^{2}$ for $5 \mathrm{~min}$. Figure 6 a shows the photos of periodontitis rat receiving the treatment of sPDMA@ICG NPs and laser irradiation.

DCFH-DA was next used as a fluorescence probe to detect the generation levels of ROS in periodontal tissues at treatment site. Figure $6 \mathrm{~b}$ shows the confocal microscope images of frozen tissue sections with DCFH-DA staining. The green fluorescence of produced DCF was only observed in the periodontal tissues of the rats receiving the treatments of free ICG and SPDMA@ICG NPs with laser irradiation, demonstrating the generations of ROS induced by these treatments. Figure $6 \mathrm{c}$ shows the corresponding quantitative analysis of DCF fluorescence intensities. It was obvious that sPDMA@ICG NPs with laser irradiation induced a much higher level of ROS generation than free ICG with laser irradiation. These results indicated that SPDMA@ICG NPs had a potent PDT performance in periodontitis rats owing to their adhesion and penetration towards the plaque biofilms and bacterial membranes.

Within 5 min of laser irradiation, the temperature changes at periodontal tissues in the rats with administration of PBS (the control) and SPDMA@ICG NPs were also detected using a thermal imaging camera. As shown in Fig.6d, sPDMA@ICG NPs increased the temperature of periodontal tissue rapidly to $43.1^{\circ} \mathrm{C}$ within $1 \mathrm{~min}$ of laser irradiation and $51.2^{\circ} \mathrm{C}$ within $5 \mathrm{~min}$ of laser irradiation. According to an investigation we previously reported [38], this temperature will not bring about the serious damages to the normal tissues. On the contrary, the temperature of periodontal tissue in the control rat did not increase significantly within this period of laser irradiation. These results indicated that sPDMA@ICG NPs also had a potent PTT performance in periodontitis rats.

\section{In vivo anti-periodontitis effects of sPDMA@ICG NPs with laser irradiation}

Periodontitis rats were used to evaluate the in vivo anti-periodontitis effects of synergistic PTT and PDT mediated by sPDMA@ICG NPs. The treatments including sPDMA, free ICG, sPDMA@ICG NPs alone and their combination with laser irradiation as described above were carried out once a week for consecutive 3 weeks. Afterwards, the resorption of alveolar bones in these treated rats was analyzed using the microcomputed tomography (micro-CT). The three-dimensional digital and tomographic images of alveolar bones were separately reconstructed with CTVox software and DataViewer software, shown in Fig. 7a and b. Compared to the negative (-) control rat, the alveolar bone resorption was very obvious in the positive (+) control rat, confirming the success construct of the periodontitis rat model. Upon laser irradiation, free ICG and sPDMA@ICG NPs both remarkably inhibited the alveolar bone resorption in periodontitis rats. By comparison, sPDMA@ICG NPs with laser irradiation exhibited a much stronger inhibitory effect on the alveolar bone resorption, and the treated rats had the alveolar bone heights similar to that of the control (-) rats. The distance between alveolar bone crest (ABC) and cement enamel junction (CEJ) was determined by the three points from the mesial to distal root surface of the second molars. 
Bone volume (BV), tissue volume (TV) and their ratios (BV/TV) around the ligated molars were further calculated by using CTAn software. Results in Fig. 7c and $d$ showed that the rats receiving the treatment of sPDMA@ICG NPs with laser irradiation had the shortest distance of CEJ-ABC and the highest of $\mathrm{BV} / \mathrm{TV}$. These results were basically consistent with the in vitro antibacterial results, confirming that sPDMA@ICG NPs can efficiently prevent the alveolar bone resorption through exerting the PTT and PDT performances.

When all treatments were completed, the rats were euthanized and their periodontitis lesions (palatal gingiva and surrounding mucosa) were removed from the left maxillary second molar for histopathological examination. The microscopic images of tissue sections stained with hematoxylin and eosin (H\&E) are shown in Fig. 8a. Different degrees of inflammatory responses (incomplete epithelium, epithelial hyperplasia, irregular arrangement of basal cells, and infiltration of inflammatory cells) were observed in the control(+) rats and the rats receiving the treatments of SPDMA with and without laser irradiation, free ICG and SPDMA@ICG NPs. However, these inflammatory responses were greatly ameliorated in the rats treated with free ICG and sPDMA@ICG NPs after laser irradiation. More importantly, sPDMA@ICG NPs with laser irradiation almost completely prevented the progress of periodontitis, which was manifested in the normal morphological and closely arranged epithelial cells as well as no obvious inflammatory changes in lamina propria.

As two of the inflammatory factors, tumor necrosis factor-a (TNF-a) and interleukin-1 $\beta$ (IL-1 $\beta$ ) play the important roles in the regulation of local inflammatory responses in periodontitis [39]. TNF-a can induce the inflammation, promote the secretion of matrix metalloproteins, activate the osteoclasts, and destroy the periodontal tissue $[40,41]$. IL-1 $\beta$ can recruit and activate the neutrophile granulocytes, improve the release of inflammatory mediators, and decompose the connective tissue [42, 43]. These inflammatory factors will be convenient for pathogenic bacteria and their toxic metabolites to invade deep tissues and promote the occurrence and development of periodontitis [44]. Therefore, we further evaluated the protein levels of TNF- $\alpha$ and IL-1 $\beta$ in tissues by immumohistochemical staining. As can be seen in Fig. 8b and $c$, sPDMA@ICG NPs with laser irradiation evidently reduced the protein levels of these two inflammatory factors as compared to the control(+) and the other treatments. It thus can be deduced that synergistic PTT and PDT mediated by sPDMA@ICG NPs relieved the inflammatory responses in periodontitis rats.

\section{Conclusions}

In this work, a nanosystem was developed through self-assembling of ICG and SPDMA in aqueous solution for synergistic PTT and PDT against periodontitis. As-prepared sPDMA@ICG NPs were regularly spherical in shape, and their particle size and zeta potential were approximately $206 \mathrm{~nm}$ and $+18.4 \mathrm{mV}$, respectively. Benefiting from the polycationic brush architecture of sPDMA, sPDMA@ICG NPs had excellent adsorption and penetration abilities for Pg and successfully delivered ICG into the bacteria cells. Upon 808 nm laser irradiation, sPDMA@ICG NPs showed synergistic PTT and PDT performances, and efficiently inhibited the in vitro growth of Pg. In periodontitis rats, sPDMA@ICG NPs effectively inhibited the alveolar bone resorption and relieved the inflammatory responses after laser irradiation. Thus, it can 
be seen that SPDMA@ICG NPs will be a promising nano-photosensitizer for synergistic PTT and PDT for antibacterial and periodontitis treatments in clinic.

\section{Declarations}

\section{Acknowledgements}

Not applicable.

\section{Authors' contributions}

$Y W, M Z$ and $C L$ designed this research and drafted the manuscript. ES and LB carried out all experiments. $\mathrm{XY}$ and $\mathrm{YW}$ analyzed the data and prepared the figures. $\mathrm{LM}$ and HW participated in the in vitro and in vivo experiments.

\section{Funding}

This research was supported by the National Natural Science Foundation of China (81972903 and 12074284), the Natural Science Foundation of Tianjin City (No. 18JCYBJC92400) and the Talent Excellence Program from Tianjin Medical University.

\section{Availability of data and materials}

All data generated or analyzed during this study are included in this article and its additional information file.

\section{Ethics approval and consent to participate}

All animal care protocols and experiments involved in this study were in accordance with protocols approved by the Tianjin Medical University Animal Care and Use Committee.

\section{Consent for publication}

All authors concur with the submission and publication of this paper.

\section{Competing interests}

The authors declare no competing interests. 


\section{Author details.}

${ }^{1}$ School of Dentistry \& Hospital of Stomatology, ${ }^{2}$ Tianjin Key Laboratory of Technologies Enabling Development of Clinical Therapeutics and Diagnostics, School of Pharmacy, No. 22 Qixiangtai Road, Heping District, Tianjin 300070, China.

${ }^{3}$ Tianjin Key Laboratory of Biomedical Materials, Institute of Biomedical Engineering, Chinese Academy of Medical Sciences \& Peking Union Medical College, No. 238 Baidi Road, Nankai District, Tianjin 300192, China

\section{References}

1. Kassebaum NJ, Bernabé E, Dahiya M, Bhandari B, Murray CJ, Marcenes W. Global burden of severe periodontitis in 1990-2010: a systematic review and meta-regression. J Dent Res. 2014;93(11):1045-53.

2. Darveau RP. Periodontitis: a polymicrobial disruption of host homeostasis. Nat Rev Microbiol. 2010;8(7):481-90.

3. Kinane DF, Stathopoulou PG, Papapanou PN. Periodontal diseases. Nat Rev Dis Primers. 2017;3:17038.

4. Hajishengallis G. Periodontitis: from microbial immune subversion to systemic inflammation. Nat Rev Immunol. 2015;15(1):30-44.

5. Munasur SL, Turawa EB, Chikte UME, Musekiwa A. Mechanical debridement with antibiotics in the treatment of chronic periodontitis: effect on systemic biomarkers-a systematic review. Int J Environ Res Public Health. 2020;17(15):5601.

6. Greenstein G. Local drug delivery in the treatment of periodontal diseases: assessing the clinical significance of the results. J Periodontol. 2006;77(4):565-78.

7. Umeda M, Takeuchi Y, Noguchi K, Huang Y, Koshy G, Ishikawa I. Effects of nonsurgical periodontal therapy on the microbiota. Periodontol 2000. 2004;36:98-120.

8. Rotundo R, Nieri M, Cairo F, Franceschi D, Mervelt J, Bonaccini D, Esposito M, Pini-Prato G. Lack of adjunctive benefit of Er:YAG laser in non-surgical periodontal treatment: a randomized split-mouth clinical trial. J Clin Periodontol. 2010;37(6):526-33.

9. Rams TE, Sautter JD, van Winkelhoff AJ. Comparative in vitro resistance of human periodontal bacterial pathogens to tinidazole and four other antibiotics. Antibiotics. 2020;9(2):68.

10. van Winkelhoff AJ, Herrera D, Oteo A, Sanz M. Antimicrobial profiles of periodontal pathogens isolated from periodontitis patients in the Netherlands and Spain. J Clin Periodontol. 2005;32(8):893-8.

11. Hu D, Zou L, Li B, Hu M, Ye W, Ji J. Photothermal killing of methicillin-resistant staphylococcus aureus by bacteria-targeted polydopamine nanoparticles with nano-localized hyperpyrexia. ACS Biomater Sci Eng. 2019;5(10):5169-79. 
12. Jiang L, Gan CR, Gao J, Loh XJ. A Perspective on the Trends and Challenges Facing Porphyrin-Based Anti-Microbial Materials. Small. 2016;12(27):3609-44.

13. Pandey RK, Goswami LN, Chen Y, Gryshuk A, Missert JR, Oseroff A, Dougherty TJ. Nature: a rich source for developing multifunctional agents. Tumor-imaging and photodynamic therapy. Lasers Surg Med. 2006;38(5):445-67.

14. Agostinis P, Berg K, Cengel KA, Foster TH, Girotti AW, Gollnick SO, Hahn SM, Hamblin MR, Juzeniene A, Kessel D, Korbelik M, Moan J, Mroz P, Nowis D, Piette J, Wilson BC, Golab J. Photodynamic therapy of cancer: an update. CA Cancer J Clin. 2011;61(4):250-81.

15. Hurdle JG, O'Neill AJ, Chopra I, Lee RE. Targeting bacterial membrane function: an underexploited mechanism for treating persistent infections. Nat Rev Microbiol. 2011;9(1):62-75.

16. Landis RF, Li CH, Gupta A, Lee YW, Yazdani M, Ngernyuang N, Altinbasak I, Mansoor S, Khichi MAS, Sanyal $A$, et al. Biodegradable nanocomposite antimicrobials for the eradication of multidrugresistant bacterial biofilms without accumulated resistance. J Am Chem Soc. 2018;140(19):617682.

17. Li Z, Pan W, Shi E, Bai L, Liu H, Li C, Wang Y, Deng J, Wang Y. A multifunctional nanosystem based on bacterial cell-penetrating photosensitizer for fighting periodontitis via combining photodynamic and antibiotic therapies. ACS Biomater Sci Eng. 2021;7(2):772-86.

18. Cherukula K, Lekshmi KM, Uthaman S, Cho K, Cho CS, Park IK. Multifunctional inorganic nanoparticles: recent progress in thermal therapy and imaging. Nanomaterials. 2016;6(4):76.

19. Zhao S, Huang W, Wang C, Wang Y, Zhang Y, Ye Z, Zhang J, Deng L. Dong A. Screening and matching amphiphilic cationic polymers for efficient antibiosis. Biomacromol. 2020;21(12):5269-81.

20. Kuroda K, Caputo GA. Antimicrobial polymers as synthetic mimics of host-defense peptides. WIREs Nanomed Nanobiotechnol. 2013;5(1):49-66.

21. Venkatesh M, Barathi VA, Goh ETL, Anggara R, Fazil M, Ng AJY, Harini S, Aung TT, Fox SJ, Liu S, et al. Antimicrobial activity and cell selectivity of synthetic and biosynthetic cationic polymers. Antimicrob Agents Chemother. 2017;61(10):e00469-17.

22. Deka SR, Sharma AK, Kumar P. Cationic polymers and their self-assembly for antibacterial applications. Curr Top Med Chem. 2015;15(13):1179-95.

23. Cha R, Li J, Liu Y, Zhang Y, Xie Q, Zhang M. $\mathrm{Fe}_{3} \mathrm{O}_{4}$ nanoparticles modified by CD-containing star polymer for MRI and drug delivery. Colloids Surf B Biointerfaces. 2017;158:213-21.

24. Chen W, Hong Y, Zhang T, Kong D, Zhang M, Zhang Q, Wang C. Star-shaped poly(2-aminoethyl methacrylate)s as non-viral gene carriers: exploring structure-function relationship. Colloids Surf $B$ Biointerfaces. 2019;181:721-7.

25. Wei H, Pahang JA, Pun SH. Optimization of brush-like cationic copolymers for nonviral gene delivery. Biomacromol. 2013;14(1):275-84.

26. Dai Z, Gjetting T, Mattebjerg MA, Wu C, Andresen TL. Elucidating the interplay between DNAcondensing and free polycations in gene transfection through a mechanistic study of linear and 
branched PEl. Biomaterials. 2011;32(33):8626-34.

27. Martello F, Piest M, Engbersen JF, Ferruti P. Effects of branched or linear architecture of bioreducible poly(amido amine)s on their in vitro gene delivery properties. J Control Release. 2012;164(3):372-9.

28. Lv H, Zhang S, Wang B, Cui S, Yan J. Toxicity of cationic lipids and cationic polymers in gene delivery. J Control Release. 2006;114(1):100-9.

29. Hajishengallis G, Darveau RP. Curtis MA The keystone-pathogen hypothesis. Nat Rev Microbiol. 2012;10(10):717-25.

30. Lamont RJ, Koo H, Hajishengallis G. The oral microbiota: dynamic communities and host interactions. Nat Rev Microbiol. 2018;16(12):745-59.

31. Zhang $M$, Xiong Q, Wang Y, Shen W, Liu L, Wang Q, Zhang Q. A well-defined coil-comb polycationic brush with "star polymers" as side chains for gene delivery. Polym Chem. 2014;5(16):4670-8(9).

32. Uchida E, Ikada Y. Topography of polymer chains grafted on a polymer surface underwater. Macromolecules. 1997;30(18):5464-9.

33. Szejtli J. Introduction and general overview of cyclodextrin chemistry. Chem Rev. 1998;98(5):174354.

34. Barros TC, Toma SH, Toma HE, Bastos EL, Baptista MS. Polymethine cyanine dyes in $\beta$-cyclodextrin solution: multiple equilibria and chemical oxidation. J Phys Org Chem. 2010;23(10):893-903.

35. Townes CL, Michailidis G, Hall J. The interaction of the antimicrobial peptide CLEAP-2 and the bacterial membrane. Biochem Biophys Res Commun. 2009;387(3):500-3.

36. Marsh PD. Dental plaque: biological significance of a biofilm and community life-style. J Clin Periodontol. 2005;32(Suppl 6):7-15.

37. Roberts AP, Mullany P. Oral biofilms: a reservoir of transferable, bacterial, antimicrobial resistance. Expert Rev Anti Infect Ther. 2010;8(12):1441-50.

38. Cheng Y, Chen Q, Guo Z, Li M, Yang X, Wan G, Chen H, Zhang Q, Wang Y. An intelligent biomimetic nanoplatform for holistic treatment of metastatic triple-negative breast cancer via photothermal ablation and immune remodeling. ACS Nano. 2020;14:15161-81.

39. Graves DT, Cochran D. The contribution of interleukin-1 and tumor necrosis factor to periodontal tissue destruction. J Periodontol. 2003;74(3):391-401.

40. Algate K, Haynes DR, Bartold PM, Crotti TN, Cantley MD. The effects of tumour necrosis factor-a on bone cells involved in periodontal alveolar bone loss; osteoclasts, osteoblasts and osteocytes. $\mathrm{J}$ Periodontal Res. 2016;51(5):549-66.

41. Zhao B. TNF and bone remodeling. Curr Osteoporos Rep. 2017;15(3):126-34.

42. Cheng R, Wu Z, Li M, Shao M, Hu T. Interleukin- $1 \beta$ is a potential therapeutic target for periodontitis: a narrative review. Int J Oral Sci. 2020;12:2.

43. Papathanasiou E, Conti P, Carinci F, Lauritano D, Theoharides TC. IL-1 superfamily members and periodontal diseases. J Dent Res. 2020;99(13):1425-34. 
44. Yucel-Lindberg T, Båge T. Inflammatory mediators in the pathogenesis of periodontitis. Expert Rev Mol Med. 2013;15:e7.

\section{Figures}
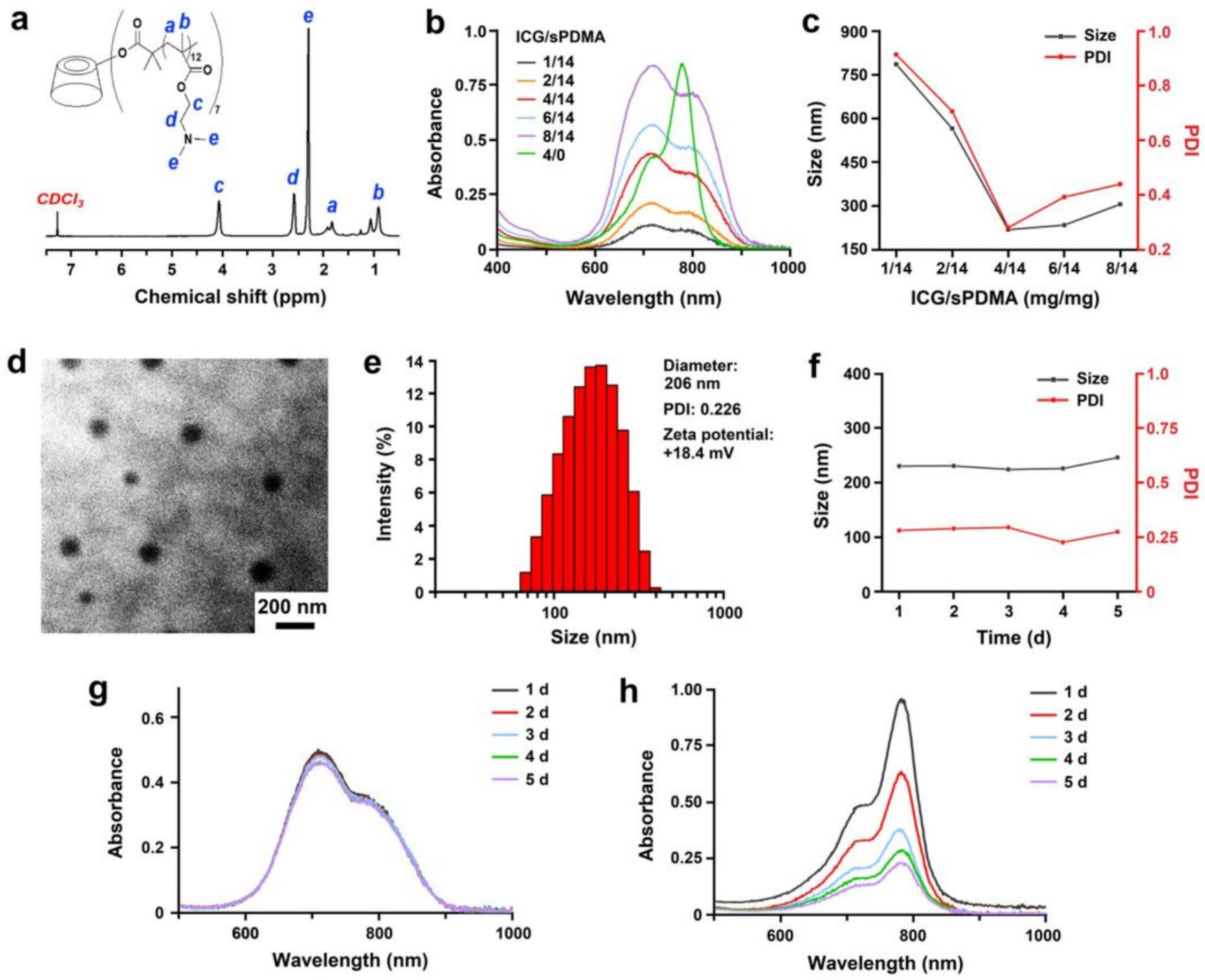

Figure 1

Preparation and characterization of sPDMA@ICG NPs. (a) Chemical structure and 1H NMR spectrum of sPDMA in CDCl3. (b) UV-Vis-NIR absorption spectra, (c) size and PDI of sPDMA@ICG NPs prepared at different weight ratios of ICG/sPDMA. (d) TEM image and (e) size distribution of sPDMA@ICG NPs at the ICG/sPDMA weight ratio of 4/14. (f) Sizes and PDIs of sPDMA@ICG NPs during storage for $5 \mathrm{~d}$. UV-VisNIR adsorption spectra of (g) sPDMA@ICG NPs and (h) free ICG in aqueous solution during storage for 5 $\mathrm{d}$ at the ICG concentration of $10 \mu \mathrm{g} / \mathrm{mL}$. 

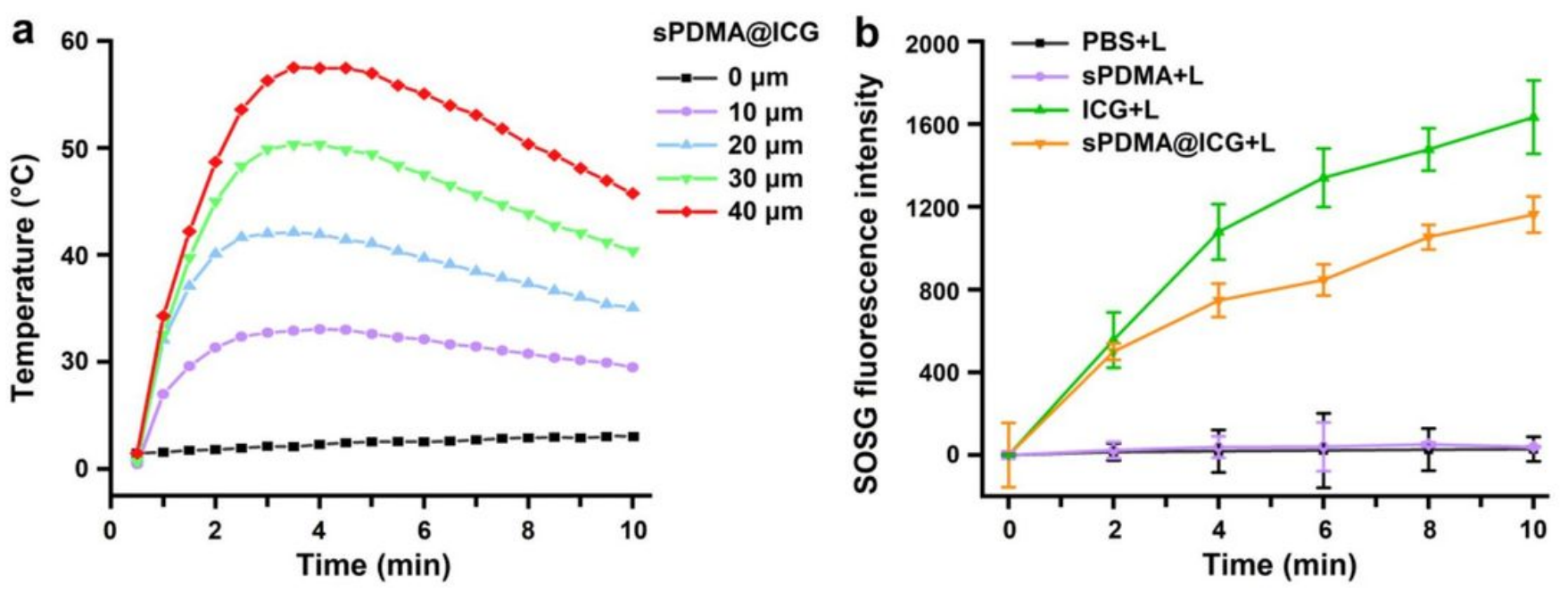

Figure 2

Photothermal and photodynamic performances of the sPDMA@ICG NP solution. (a) Temperature changes of the sPDMA@ICG NPs solutions upon laser irradiation at different ICG concentrations. (b) Fluorescence intensities of SOSG $(\lambda e x=504 \mathrm{~nm}, \lambda \mathrm{em}=525 \mathrm{~nm})$ in the solutions of PBS, sPDMA, free ICG and SPDMA@ICG NPs upon laser irradiation $(+\mathrm{L})$. Here, the ICG concentrations for free ICG and sPDMA@ICG NPs were $10 \mu \mathrm{g} / \mathrm{mL}$. In the above experiments, the laser irradiation was carried at $808 \mathrm{~nm}$ at $2 \mathrm{~W} / \mathrm{cm} 2$ for $10 \mathrm{~min}$. 


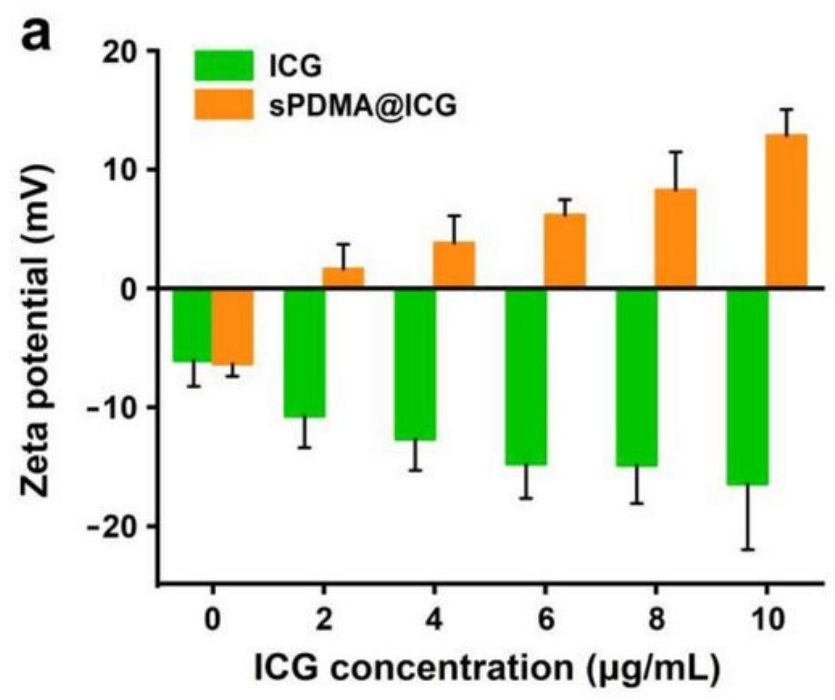

C

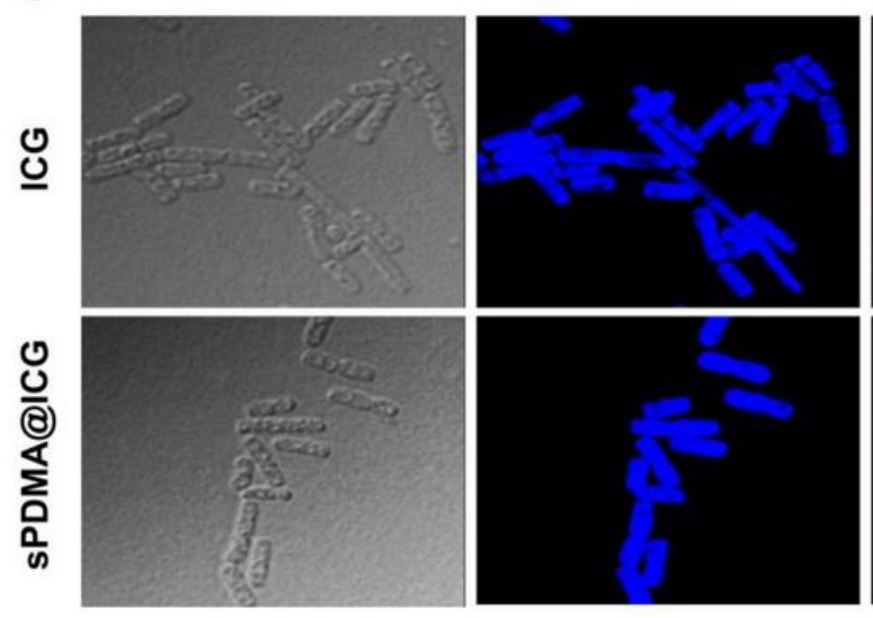

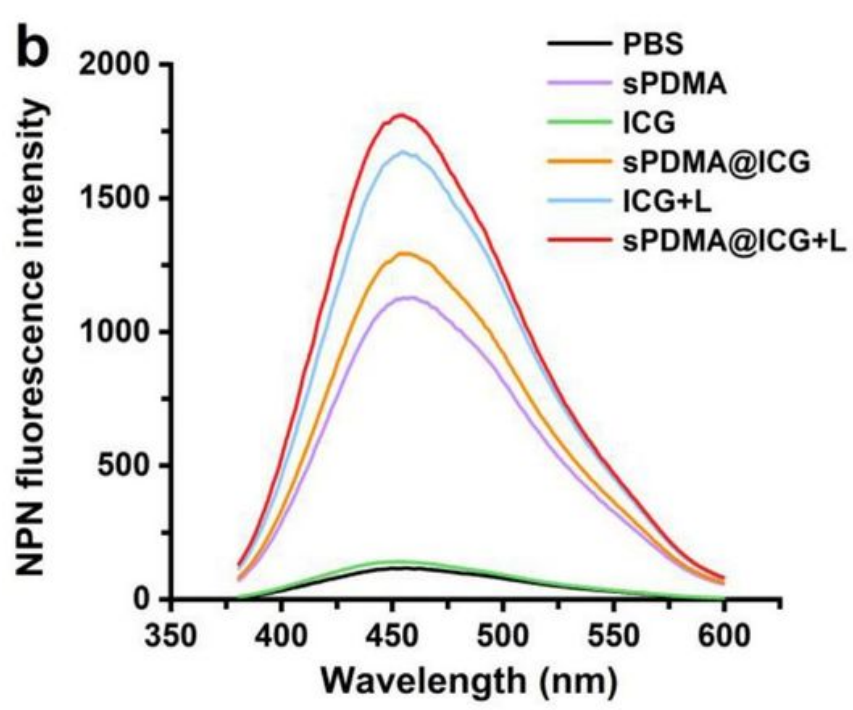

ICG
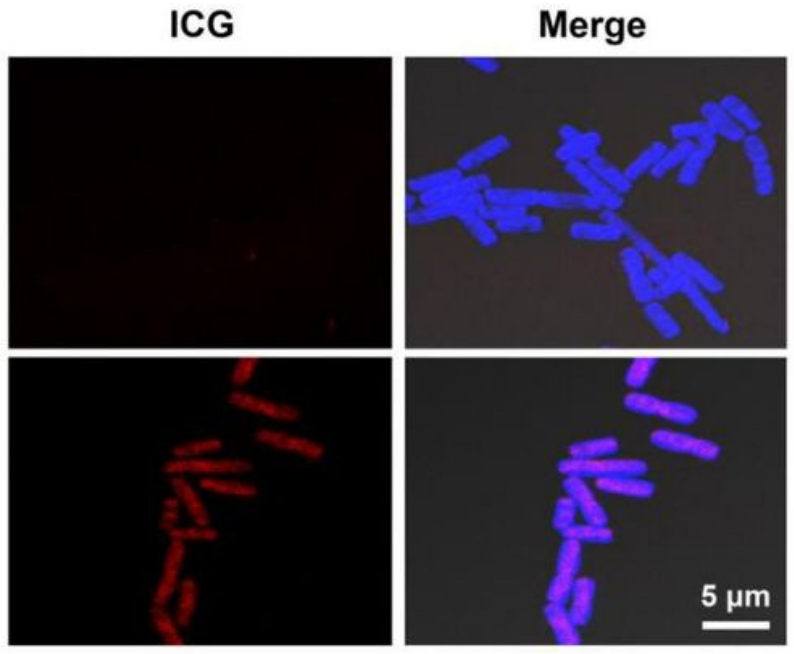

Figure 3

Adsorption and penetration abilities of sPDMA@ICG NPs towards Pg. (a) Zeta potentials of bacteria incubated with free ICG and SPDMA@ICG NPs for $3 \mathrm{~h}$ at different ICG concentrations. (b) Fluorescence emission spectra of NPN in bacteria suspensions processed with PBS, SPDMA, free ICG with and without laser irradiation, and SPDMA@ICG NPs with and without laser irradiation. Here, the laser irradiation (808 $\mathrm{nm}, 2 \mathrm{~W} / \mathrm{cm} 2$ and $5 \mathrm{~min}$ ) was carried at $3 \mathrm{~h}$ after incubation. (c) Confocal images of bacteria with incubation of free ICG and sPDMA@ICG NPs for 3 h. Blue and red fluorescence represent DAPI and ICG, respectively. 

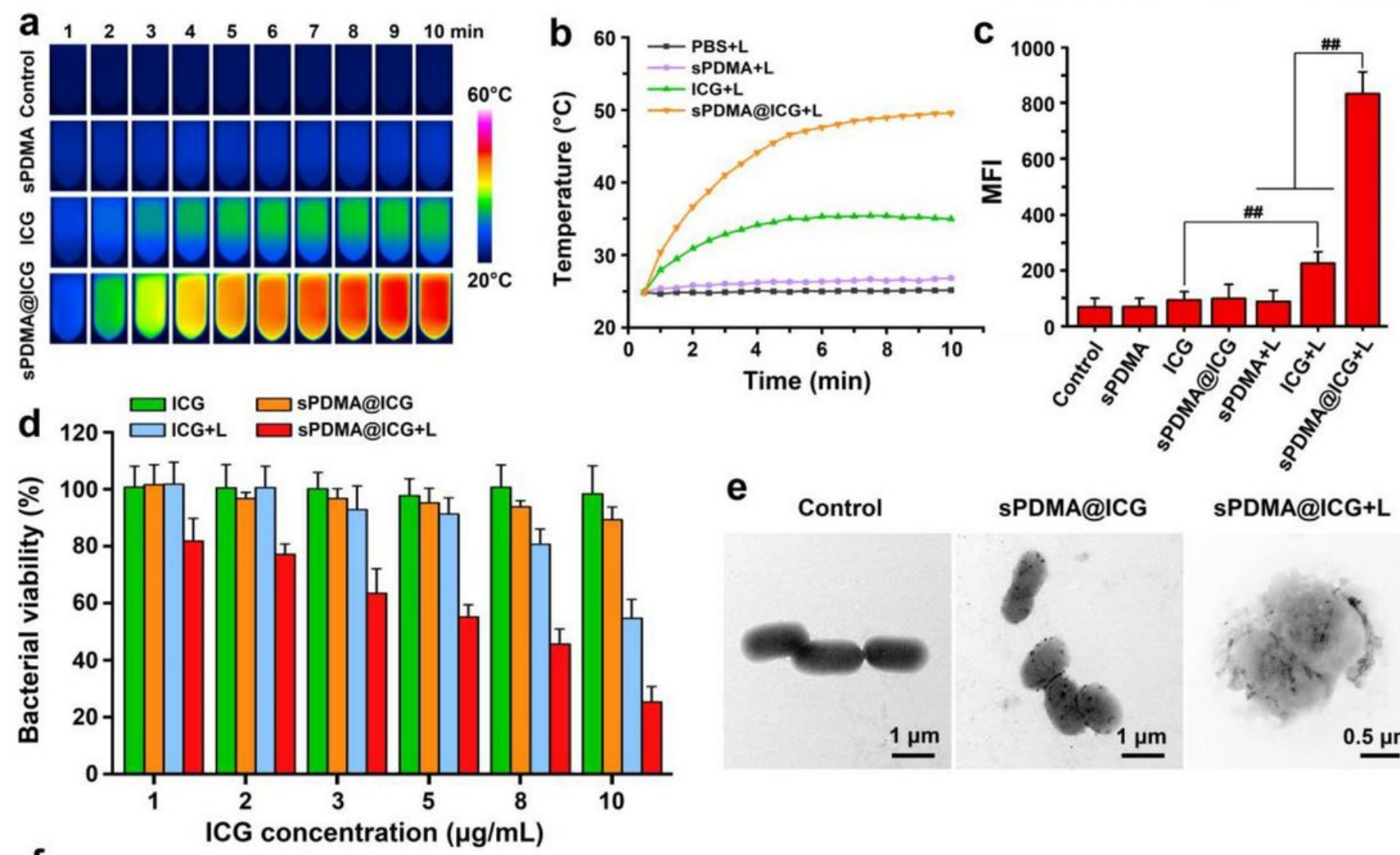

SPDMA@ICG+L

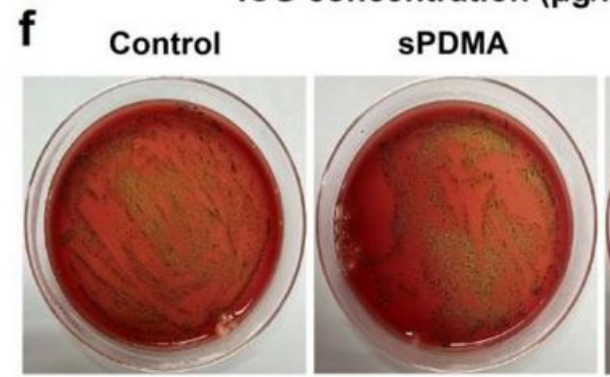

ICG
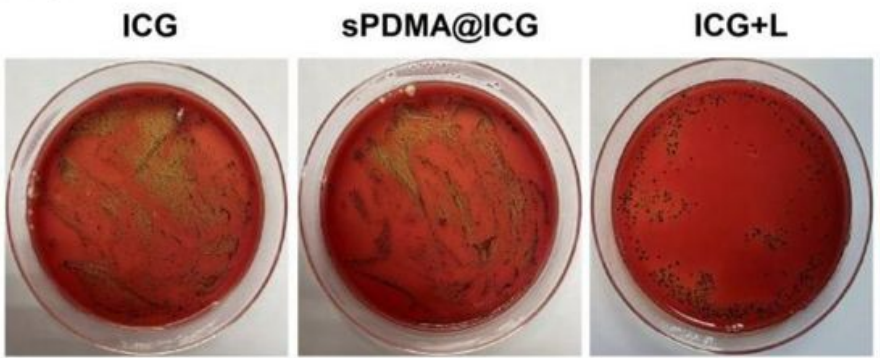

sPDMA@ICG+L

\section{Figure 4}

Antibacterial effects of sPDMA@ICG NPs with laser irradiation in Pg. (a) Infrared thermal images and (b) temperature changes of bacterial suspensions incubated with PBS, sPDMA, free ICG and SPDMA@ICG NPs for $3 \mathrm{~h}$, followed by laser irradiation. (c) Mean fluorescence intensities (MFI) of DCF in bacterial suspensions after various processions. (d) Antibacterial activities of free ICG and sPDMA@ICG NPs with and without laser irradiation at different ICG concentrations. (e) TEM images of bacteria after processions of PBS (the control), sPDMA@ICG NPs with and without laser irradiation. (f) Photos of bacterial colonies after various processions. In the experiments for (a) (b) (c) (e), and (f), the ICG concentration was $10 \mu \mathrm{g} / \mathrm{mL}$ and the laser irradiation was carried at $808 \mathrm{~nm}$ at $2 \mathrm{~W} / \mathrm{cm} 2$ for $10 \mathrm{~min}$. \#\# indicates $p<0.01$ for comparison between two groups. 


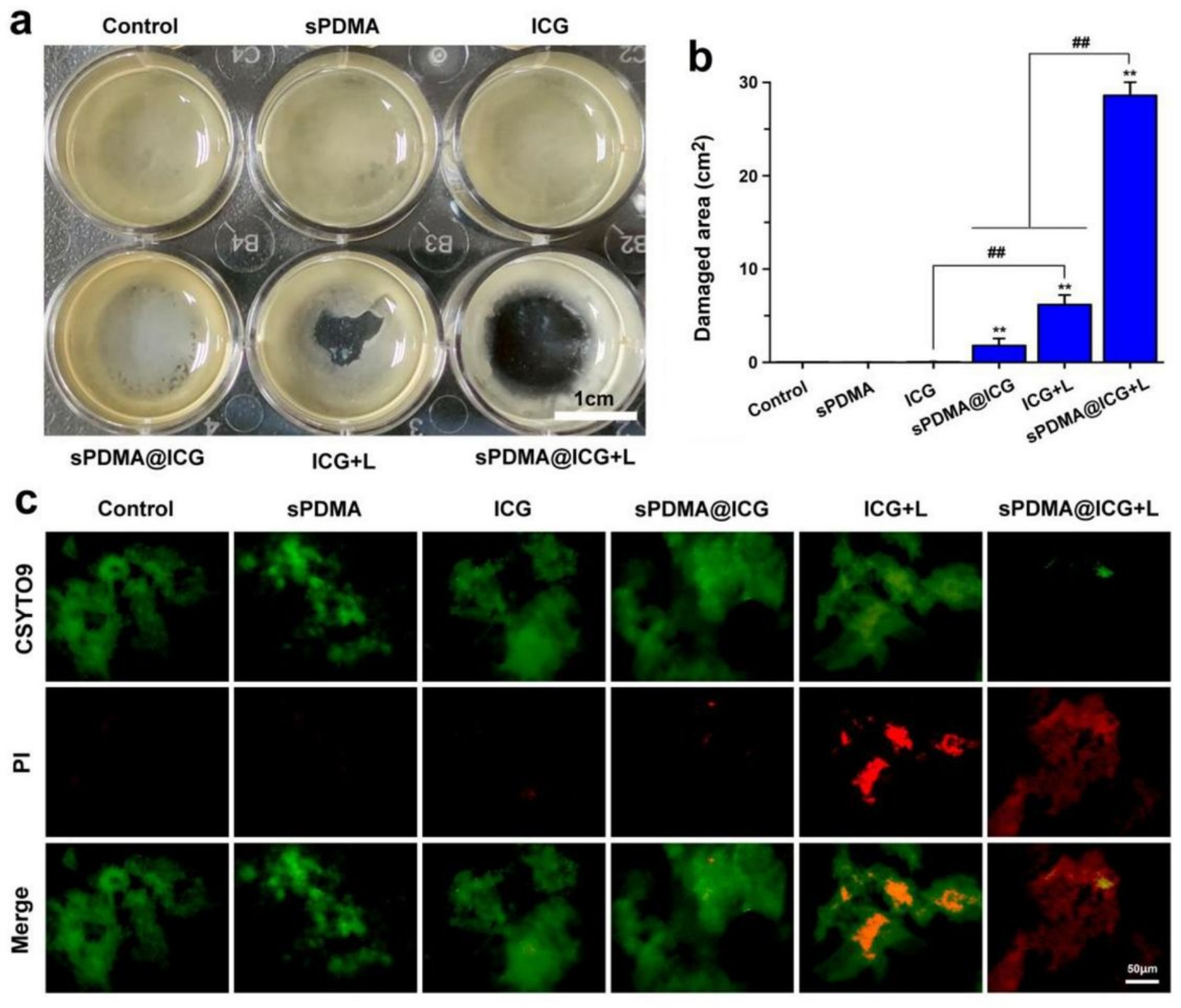

\section{Figure 5}

Destruction effect of sPDMA@ICG NPs with laser irradiation on plaque biofilm. (a) Photos of plaque biofilms after various processions and (b) comparison for destructive area analyzed using Image J. (c) Fluorescence microscopic images of plaque biofilms stained with the Live/Dead staining kit after various processions. In these above experiments, the plaque biofilms were formed by pathogenic bacteria sourced from periodontitis rats. Here, Plaque biofilms were incubated with sPDMA, ICG and SPDMA@ICG NPs for $3 \mathrm{~h}$, and afterwards, laser irradiation was carried out at $808 \mathrm{~nm}$ at $2 \mathrm{~W} / \mathrm{cm} 2$ for $5 \mathrm{~min}$. ** indicates $p<0.01$ compared to the control; \#\# indicates $p<0.01$ for comparison between two groups. 

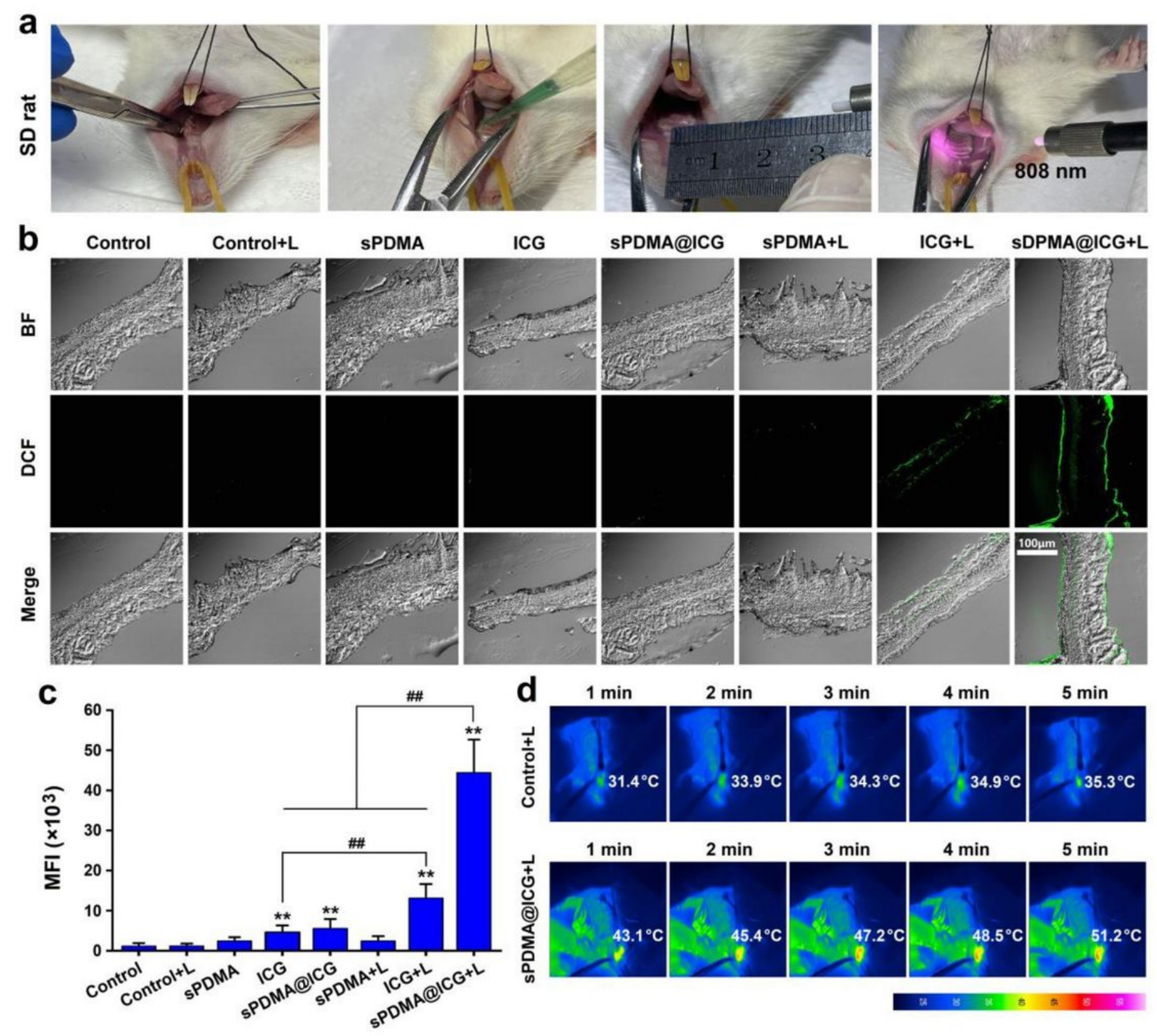

\section{Figure 6}

PTT and PDT performances of sPDMA@ICG NPs in periodontitis rats. (a) Photos of periodontitis rat with administration of sPDMA@ICG NPs and laser irradiation. (b) Confocal microscopic images of DCFH-DA stained tissue sections sourced from periodontitis lesions in the rats after treatments of SPDMA, ICG and sPDMA@ICG NPs with or without laser irradiation. (c) Comparison for the mean fluorescence intensities of produced DCF in periodontal tissues. (d) Infrared thermal images of periodontal tissues during laser irradiation after administration of PBS (the control) and sPDMA@ICG NPs. In these experiments, the laser irradiation ( $808 \mathrm{~nm}, 2 \mathrm{~W} / \mathrm{cm} 2,5 \mathrm{~min}$ ) was carried out locally at the periodontitis site. ${ }^{* \star}$ indicates $p<0.01$ compared to the control $(+)$; \#\# indicates $p<0.01$ for comparison between two groups. 

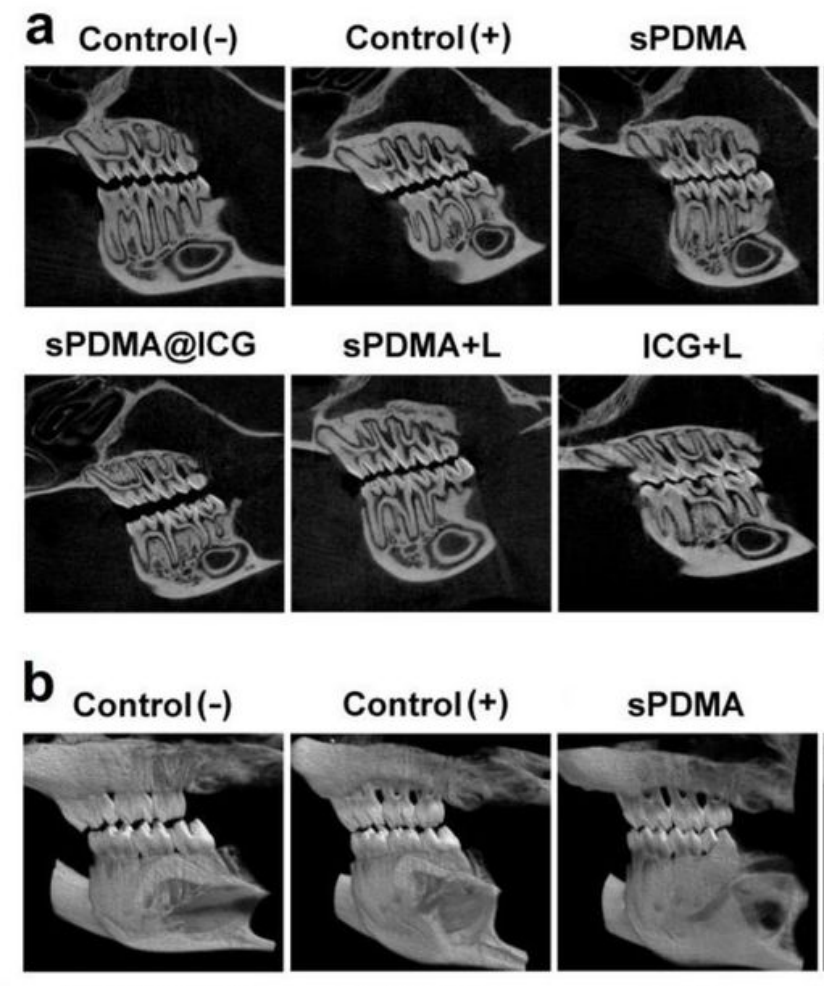

sPDMA@ICG
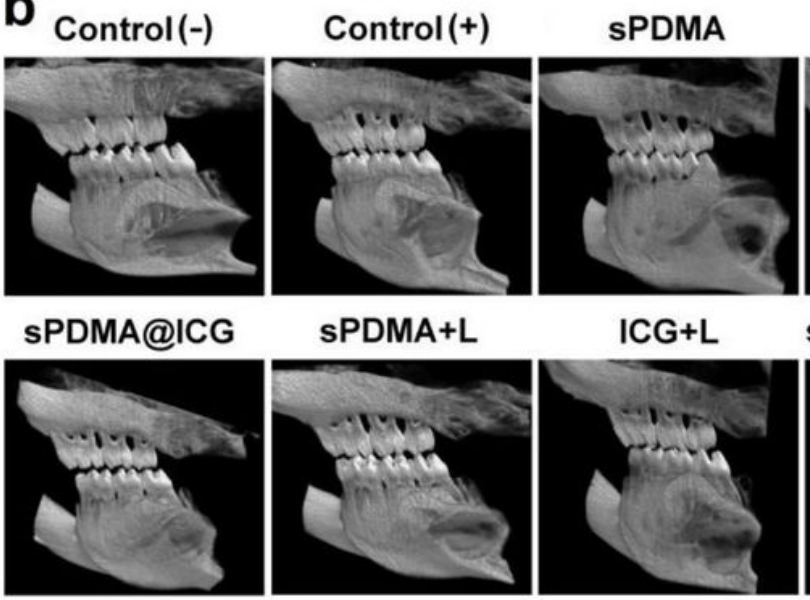

sPDMA@ICG+L
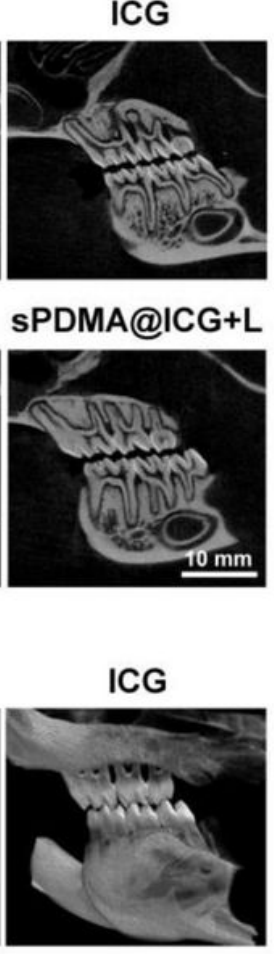

sPDMA@ICG+L

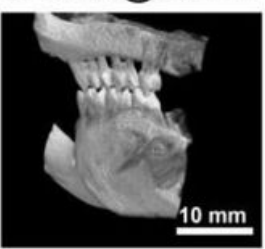

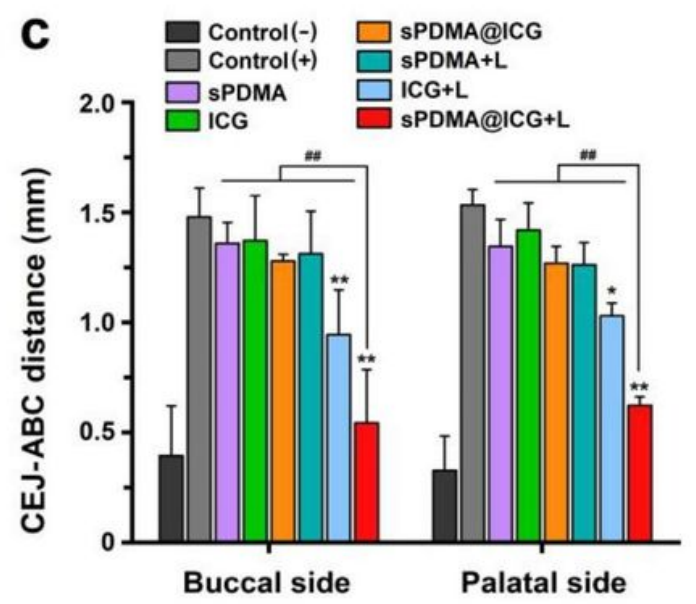

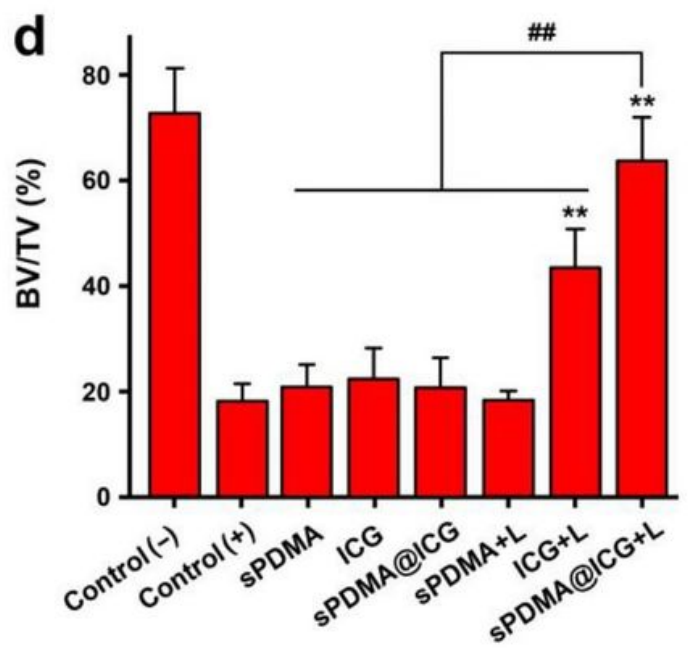

Figure 7

Inhibitory effect of sPDMA@ICG NPs with laser irradiation on the alveolar bone resorption in periodontitis rats. (a) Cross-sectional and (b) three-dimensional micro-CT images of the alveolar bones in the control rats (negative and positive) and the rats receiving the treatments of SPDMA, free ICG, sPDMA@ICG NPs with and without laser irradiation. (c) CEJ-ABC distance on the buccal and palatal sides and (d) BV/TV calculated from micro-CT results. * and $* *$ indicate $\mathrm{P}<0.05$ and $\mathrm{P}<0.01$ compared separately to the control $(+)$; \#\# indicates $p<0.01$ for comparison between two groups. 


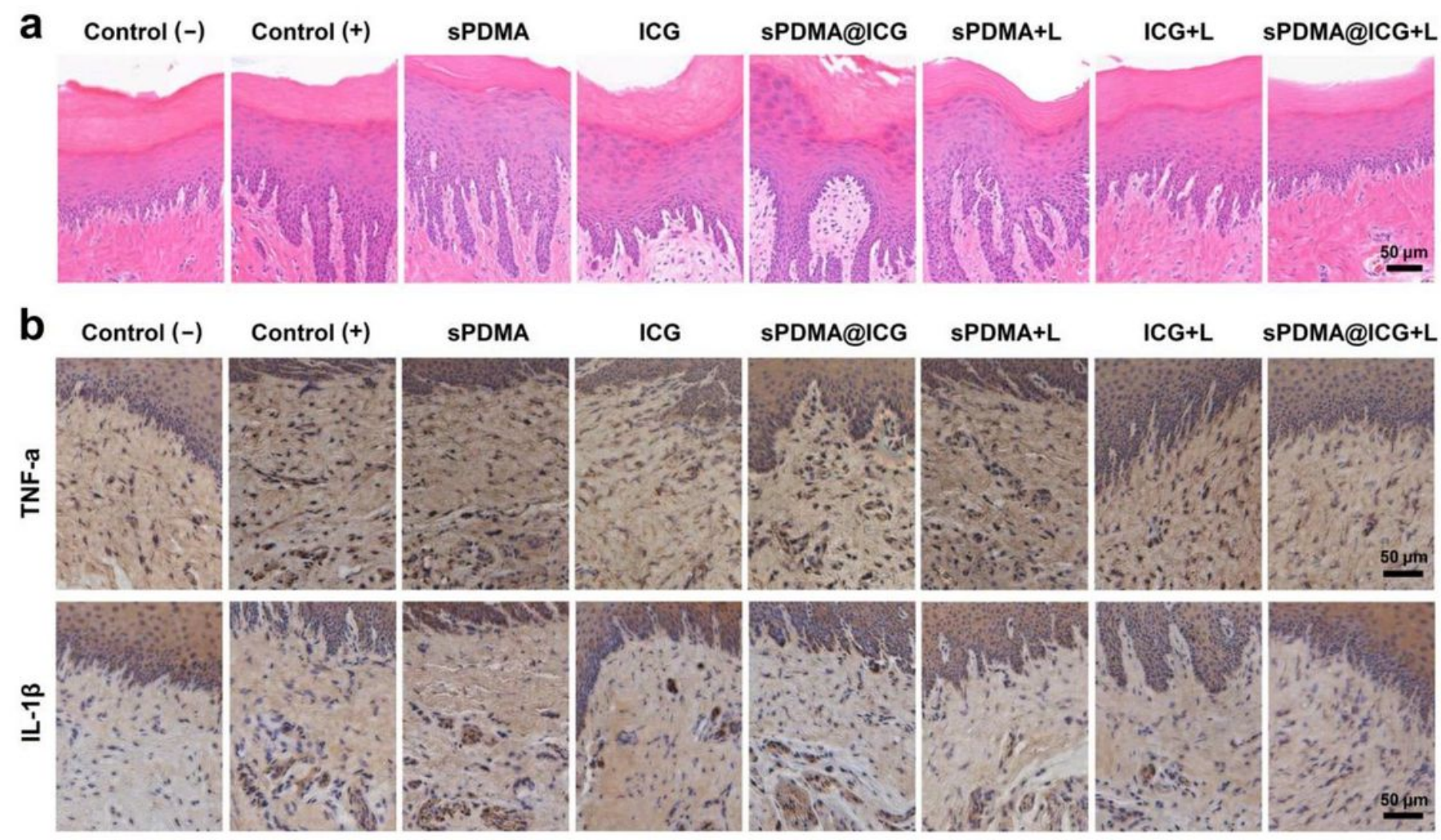

Figure 8

Microscopic images of tissue sections with staining of H\&E (a), TNF- $a$ and IL-1 $\beta$ antibodies (b) from the control rats (negative and positive) and the rats receiving the treatments of SPDMA, free ICG, sPDMA@ICG NPs with and without laser irradiation. 


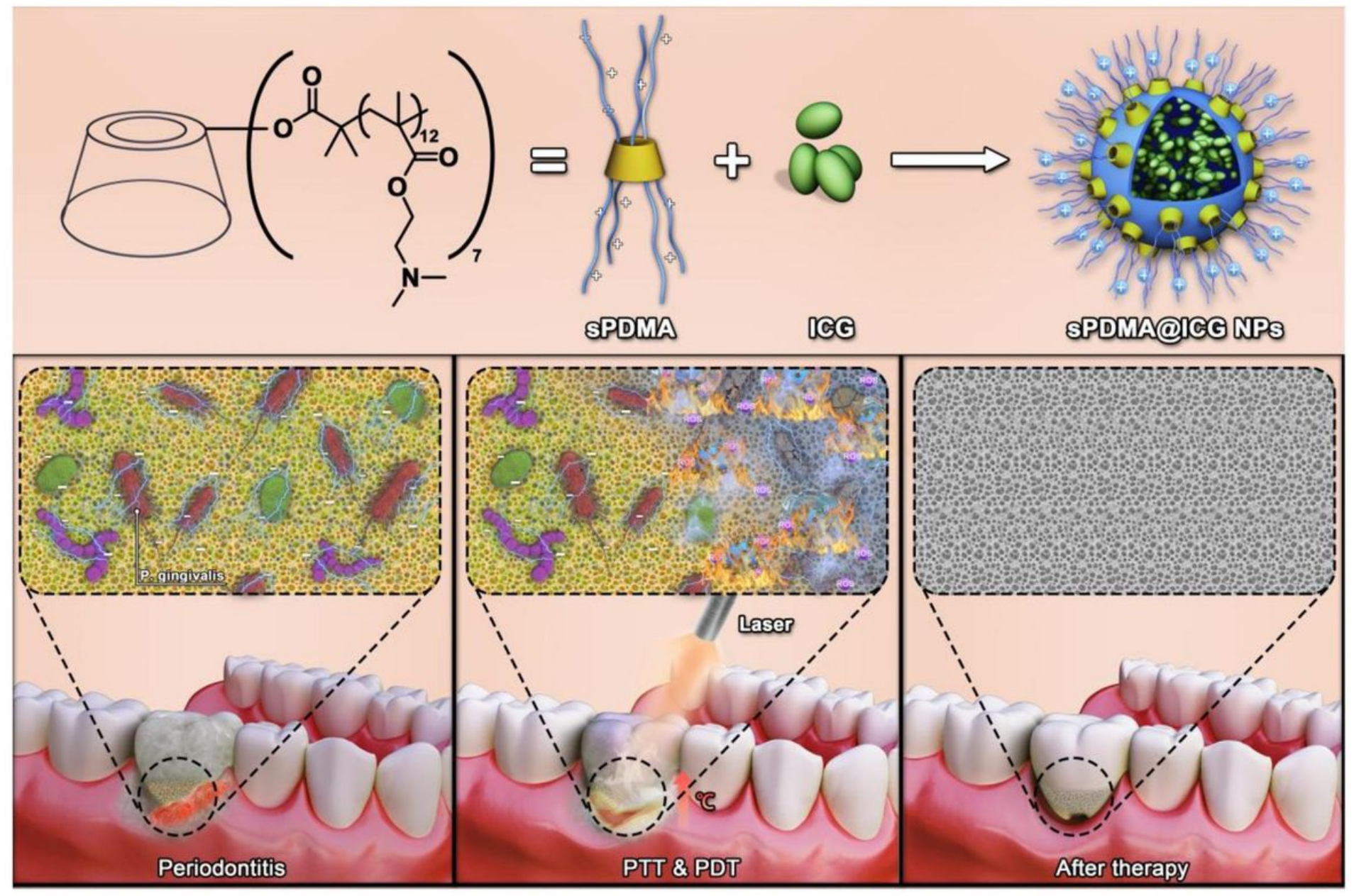

Figure 9

Scheme 1. Illustrations for preparation of sPDMA@ICG NPs and treatment progress of synergistic PTT and PDT on periodontitis.

\section{Supplementary Files}

This is a list of supplementary files associated with this preprint. Click to download.

- GraphicalAbstract.tif

- Supportinglnformation.doc 